\title{
Evidence of meaningful levels of Trypanosoma cruzi in platelet concentrates from seropositive blood donors
}

\author{
Beatriz Cancino-Faure, ${ }^{1}$ Roser Fisa, ${ }^{1}$ Cristina Riera, ${ }^{1}$ Ibeth Bula,${ }^{1}$ Enrique Girona-Llobera, ${ }^{2,3}$ \\ and Teresa Jimenez-Marco ${ }^{2,3}$
}

\begin{abstract}
BACKGROUND: According to the reported cases of transfusion-acquired Trypanosoma cruzi infection, the risk of $T$. cruzi transfusion transmission appears to be higher with platelet (PLT) products than with other blood components. The aim of this study was to investigate by quantitative real-time polymerase chain reaction (qPCR) the parasitic load detected in leukoreduced plasma and PLT concentrates collected by apheresis from seropositive T. cruzi blood donors and compare them with peripheral whole blood (WB).
\end{abstract}

STUDY DESIGN AND METHODS: During 2011 to 2013, a prospective study was carried out in a group of blood donors originating from Chagas-endemic areas but who are now living on the island of Majorca, Spain. Leukoreduced plasma and PLT concentrates were collected by apheresis from seropositive blood donors with detectable parasitemias in peripheral WB.

RESULTS: Seropositivity was found in 23 of 1201 donors studied (1.9\%), and T. cruzi DNA with less than 1 parasite equivalent $/ \mathrm{mL}$ was detected in peripheral WB in $60.86 \%$ (14 of 23 ) of these. The study in blood components obtained by apheresis from these donors showed that $T$. cruzi DNA with a mean \pm SD parasitic load of $5.33 \pm 6.12$ parasite equivalents $/ \mathrm{mL}$ was detected in $100 \%$ of the PLT concentrate samples. Parasite DNA was undetectable in the extract taken from plasma collected from donors with a positive qPCR in peripheral WB.

CONCLUSION: The higher parasitic load found in PLT concentrates compared to plasma and peripheral WB would explain the higher transfusion transmission risk of Chagas disease associated with PLT transfusions described in the reported cases of transfusion-acquired T. cruzi infection.
A $s$ a result of globalization and immigration, emerging blood-borne pathogens such as Trypanosoma cruzi, the causal agent of Chagas disease, are gaining importance in nonendemic countries. $^{1-3}$ The 20 cases associated with transfusion transmission of T. cruzi reported in North America and Spain between 1987 and 2011 were all related to platelet (PLT) concentrates or whole blood (WB), some of which had even been leukoreduced and irradiated. ${ }^{4}$ In these cases, transfusion transmission was confirmed using hemoculture, polymerase chain reaction (PCR), or serology to study the presence of parasites in blood from transfused patients. The retrospective study of the blood component or WB donors involved in these donations was in all cases carried out by serology and, additionally in some cases, using PCR to search for parasite DNA. No

ABBREVIATIONS: BIBB = Balearic Islands Blood Bank; $\mathrm{Cq}=$ quantification cycle; GEB = guanidine-EDTA-whole blood; qPCR = quantitative real-time polymerase chain reaction; $\mathrm{WB}=$ whole blood.

From the ${ }^{1}$ Laboratori de Parasitologia, Departament de Microbiologia i Parasitologia Sanitàries, Facultat de Farmàcia, Universitat de Barcelona, Barcelona; the ${ }^{2}$ Fundació Banc de Sang i Teixits de les Illes Balears, Mallorca, Balearic Islands; and the ${ }^{3}$ Institut Universitari d' Investigació en Ciències de la Salut (IUNICS), Universitat de les Illes Balears, Mallorca,

Balearic Islands, Spain

Address reprint requests to: Dr Roser Fisa, Av. Joan XXIII s.n. E-08028, Barcelona, Spain; e-mail: rfisa@ub.edu.

This work is part of a research study supported by the National R\&D+i Plan 2008-2011 and ISC III -Subdirección General de Evaluación y Fomento de la Investigación (PI 10/ 00533), was in part funded by CONICYT/Becas Chile, and is part of the Generalitat de Catalunya 2014 SGR 1241 program.

Received for publication September 22, 2014; revision received November 24, 2014; and accepted November 24, 2014.

doi:10.1111/trf.12989

(C) $2015 \mathrm{AABB}$

TRANSFUSION 2015;00;00-00 
parasite cultures or PCR DNA amplifications were performed in the units that had already been transfused. ${ }^{5-8}$

As T. cruzi is present in the human bloodstream as a trypomastigote, the extracellular form of T. cruzi, experimental assays have suggested that as T. cruzi adheres to leukoreduction filter fibers, such filters could be used as a means of reducing this organism in infected blood. ${ }^{9,10}$ However, the efficacy of leukoreduction to remove parasites from red blood cell (RBC) units has not been studied and it is not known whether this method completely eliminates the risk of transfusion transmission.

Even though all blood components are potentially infectious, there is no empiric evidence in the literature that RBCs or plasma units are implicated in the transfusion transmission of T. cruzi. Most of the reported cases have been associated with PLT transfusion. ${ }^{5-8,11}$

Over the past few decades, innovative devices, new technologies, and new techniques have been developed in an attempt to decrease the risk of pathogen transfusion transmission, as well as to reduce adverse reactions to transfusion, without altering the quality of blood components. One of these techniques is the leukoreduction of blood products using centrifugation, elutriation, or filtration, based on size, density, or stickiness of the white blood cells (WBCs). The generally accepted clinical benefits include prevention of febrile reactions, alloimmunization to HLAs, and transmission of infectious agents, as well as pathogens associated with WBCs, such as cytomegalovirus or Epstein-Bar virus. ${ }^{12}$

During 2011 through 2013, a prospective study was carried out in blood donors who, although living on the Island of Majorca, originated from Chagas-endemic areas. T. cruzi serologic screening was performed to identify positive donors and determine the seropositivity in our geographical area and to enable their inclusion in a qualitative and quantitative parasite study. The parasitic load in PLTs and plasma concentrated obtained by apheresis was studied by quantitative real-time PCR (qPCR) and compared with the parasitemia in peripheral WB.

The aim of this study was to investigate the parasite loads present in WB and other blood components, such as plasma and PLTs, from seropositive T. cruzi blood donors and determine whether or not there are any differences in parasitemia levels that could explain which component presents the greatest risk of transmitting pathogens through transfusion.

\section{MATERIALS AND METHODS}

\section{Blood donors}

During 2011 through 2013, a total of 1201 blood donors who originated from Chagas-endemic areas, but are now living in the Balearic Islands, were tested for T. cruzi-specific antibodies in the Balearic Islands Blood Bank (BIBB).
All donors with positive serology who had not been previously treated for the parasite, and consented to be included in the study, were analyzed for parasite load in peripheral WB and blood components, plasma, and PLTs obtained by apheresis. All of them were permanently rejected as donors for patient transfusions.

\section{Ethics statement}

This study was approved by the ethical committees of the participating institutions, the Balearic Island Ethic Committee and the Research Ethics Committee of the University of Barcelona. Written informed consent was obtained from all donors for the screening and apheresis study.

In accordance with the Spanish and European regulations, our standard operating procedure for donors who were seropositive for $T$. cruzi by the screening test at the blood bank involved permanent deferral for these individuals. Additionally, donors were immediately referred to the Infectious Disease Unit at the University Hospital in Majorca for follow-up and treatment. Treatment was never delayed by participation in this study.

\section{Screening study}

Five milliliters of serum was obtained for serologic study and $10 \mathrm{~mL}$ of peripheral $\mathrm{WB}$ was collected and immediately mixed with an equal volume of $6 \mathrm{~mol} / \mathrm{L}$ guanidine hydrochloride- $0.2 \mathrm{~mol} / \mathrm{L}$ EDTA buffer (Sigma, St Louis MO) for molecular study. The mixture guanidine-EDTAWB (GEB) was stored at $4^{\circ} \mathrm{C}$ until the DNA purification. GEB samples were not boiled. ${ }^{13}$

\section{Apheresis study}

\section{Cell separators and procedures}

Fenwal Amicus Version 3.11. Fenwal Amicus (Fresenius Kabi, Bad Homburg, Germany) is an apheresis device capable of dual- and single-needle plateletpheresis. The PLT-rich plasma is separated from the RBCs and WBCs in a bell-shaped chamber with two compartments. After the elutriation process, PLT-rich plasma is pumped to the collection chamber as so-called dry PLTs, that is, highconcentration plasma-reduced PLTs. At the end of the process, the PLTs are manually resuspended by shaking and adding PLT-poor plasma and then transferred to the storage bags with additive solution (AS) at a ratio of approximately $35 \%$ plasma and $65 \%$ AS.

Two Amicus devices, Software Version 3.11, set code REF R4R2337, were used for the study. All procedures used a WB:ACD ratio of 11:1 (i.e., 1 part ACD per 11 parts WB) and citrate infusion flow of $1.35 \mathrm{~mL} / \mathrm{kg} / \mathrm{min}$. The maximum inlet and return rates were 110 and $120 \mathrm{~mL} / \mathrm{min}$, respectively.

Trima Accel Version 5.22. Trima Accel (Terumo BCT, Lakewood, CO) is a single-needle, continuous-separation apheresis device. Trima Accel Version 5 has a single-stage 
separation channel with all of the blood components flowing in one direction. The blood components separate into layers over the entire circumference according to specific gravity. PLTs are harvested from the component layer between RBCs and plasma, leukoreduced with the process-controlled leukoreduction system, and collected in the final storage container.

Three Trima Accel machines, Version 5.22, Set Code REF 80420, were used for the study. All procedures used a WB:ACD ratio of 11:1 (i.e., 1 part ACD per 10 parts WB) and maximum citrate infusion flow of $1.1 \mathrm{~mL} / \mathrm{min} / \mathrm{L}$ total blood volume. The maximum inlet and return rates were 100 and $140 \mathrm{~mL} / \mathrm{min}$, respectively. The PLT concentrates were collected in small amounts of plasma and diluted in AS at the end of the procedure to maintain a ratio of approximately $35 \%$ plasma and $65 \%$ AS.

Haemonetics MCS+. The Haemonetics MCS+ system (Haemonetics Corp., Braintree, MA) uses intermittentflow centrifugation to collect plasma, PLTs, WBCs, or RBCs. The grenade-shaped plasmapheresis rotation bowl is used for plasma collection. WB enters the bowl through the stationary tube and forms vertical layers of plasma and RBCs. As the bowl fills, packed cells accumulating on the outer wall displace the plasma inward, pushing it out through the effluent tube. When the bowl is full of RBCs, it must be stopped and emptied before the process can be repeated.

Two Haemonetics MCS+ apheresis machines, Set Code REF 792 P, PLT-poor plasma, were used for the study. All procedures used a WB:ACD ratio of 12:1 (i.e., 1 part ACD per 11 parts $\mathrm{WB})$. The maximum inlet and return rates were 100 and $150 \mathrm{~mL} / \mathrm{min}$, respectively.

\section{Preparations of blood components}

Plateletpheresis and PLT-plasmapheresis donations were performed on selected donors using Trima and Amicus automatic cell separators. The mean PLT unit volume collected in the plateletpheresis procedures was $300 \mathrm{~mL}$. PLT components containing $3.5 \times 10^{11}$ were suspended in approximately $35 \%$ plasma and $65 \%$ PLT AS (PAS IIIM; Terumo BCT). The mean volume of plasma unit collected in the PLT-plasmapheresis procedures was $300 \mathrm{~mL}$. All PLT and plasma components collected were leukoreduced by in-process leukoreduction (leukoreduction system chamber for Trima and elutriation for Amicus).

Plasmapheresis donations were carried out using the Haemonetics MCS +. The mean plasma volume collected was $600 \mathrm{~mL}$, and leukoreduction was performed using a leukoreduction plasma filter (Plasmaflex Plas 4, MacoPharma, Mouvaux, France) at the end of the collection procedure.

A total of eight apheresis procedures were performed from which six plasma and six leukoreduced PLT concentrates were obtained. Three blood donors with detectable parasitemia in peripheral WB consented to donate blood components by apheresis to study the parasite load. Each blood donor included in the study gave blood components in different donations. The first underwent one plateletpheresis procedure; the second one plasmapheresis and two PLT-plasmapheresis procedures; and the third one plasmapheresis, one plateletpheresis, and two PLTplasmapheresis procedures. Within the apheresis samples, ten milliliters of GEB was obtained to determine the basal parasitic load (Table 2).

Samples were collected in the BIBB and sent, no more than 2 days after the donation, to the Parasitology Laboratory of the Universitat de Barcelona for molecular analysis. The samples of plasma, PLTs, and GEB were sent at $-20^{\circ} \mathrm{C}$, at room temperature and $4^{\circ} \mathrm{C}$, respectively, and once received were immediately processed for DNA extraction.

\section{Serologic study}

The serologic study for detection of specific T. cruzi antibodies was performed by an enzyme-linked immunosorbent assay (ELISA) test for qualitative detection of total antibodies to T. cruzi in human serum or plasma (BioELISA Chagas, Biokit, Werfen Group, Barcelona, Spain) with recombinant antigen of T. cruzi and another commercially available ELISA test system (Ortho Clinical Diagnostics, Raritan, NJ) with antigen of a total extract of epimastigotes from T. cruzi in BIBB. Confirmation was performed in the Parasitology Laboratory of the Universitat de Barcelona by a third diagnostic method using Western blot with antigen of a total extract of epimastigotes from the T. cruzi Maracay strain. ${ }^{14}$

\section{Molecular study}

DNA extraction

For each sample, DNA was extracted in triplicate (Table 2) from different samples: $200 \mu \mathrm{L}$ of GEB from all seropositive donors (screening sample), $200 \mu \mathrm{L}$ of GEB at the moment of apheresis (basal sample), $200 \mu \mathrm{L}$ of PLT concentrate, and $200 \mu \mathrm{L}$ of plasma concentrate (the last two obtained by apheresis). The extraction was performed with a PCR template preparation kit (High Pure, Roche, Mannheim, Germany) and eluted in $200 \mu \mathrm{L}$ of elution buffer according to the manufacturer's instructions. The eluate was stored at $-20^{\circ} \mathrm{C}$ for qPCR analysis. To build the standard curve for quantification of parasitic loads, DNA from a culture of epimastigotes of T. cruzi (Maracay strain, $1 \times 10^{5}$ parasite $/ \mathrm{mL}$ ) was extracted in the same way as previously reported.

\section{qPCR assay}

Five microliters of extracted DNA was amplified in triplicate by qPCR (Table 2) in a qPCR instrument (LightCycler 480 , Roche) device. The primers, probes, and conditions of 


\begin{tabular}{|c|c|c|c|c|c|}
\hline & Argentina & Ecuador & Bolivia & Venezuela & Total \\
\hline Donors & 649 & 362 & 106 & 84 & 1201 \\
\hline Percentage of donors ${ }^{*}$ & 1.42 & 0.78 & 0.24 & 0.19 & 0.66 \\
\hline Seropositive donors $\dagger$ & $4(0.61)$ & $1(0.27)$ & $17(16.03)$ & $1(1.19)$ & $23(1.91)$ \\
\hline Presence of $T$. cruzi DNA† & $3(75)$ & $0(0)$ & $10(58.8)$ & $1(100)$ & $14(60.86)$ \\
\hline
\end{tabular}

the technique were as described by Piron and coworkers ${ }^{15}$ with some modifications. Briefly, the following were used, primers Cruzi 1 and Cruzi 2 and probe Cruzi 3, which was labeled with 6-carboxyfluorescein and minor groove binder. The final concentrations in the PCR mixture were as follows: $1 \times$ LightCycler 480 Probes Master (Roche), 750 $\mathrm{nmol} / \mathrm{L}$ each T. cruzi primer, and $250 \mathrm{nmol} / \mathrm{L}$ for the T. cruzi probe in a $20-\mu \mathrm{L}$ reaction volume. Detection of the RNase $\mathrm{P}$ human gene $(0.3 \times$ RNase $\mathrm{P}$ detection reagents, Life Technologies, Austin, TX) was included as an internal control of amplification in multiplex qPCR, and a nontemplate sample and DNA from seronegative human blood were included in each run as a negative control.

A sample was considered valid when the internal control was efficiently amplified and was considered positive when the quantification cycle $(\mathrm{Cq})$ was less than or equal to 40 and if at least three of the nine replicates studied for each sample were amplified. ${ }^{16}$ A standard curve was constructed with 1-in-10 serial dilutions of total DNA extracted from Maracay strain $\left(1 \times 10^{5}-0.1\right.$ parasite equivalents $/ \mathrm{mL}$ ). The parasitic load of every sample was calculated using the LightCycler 480 software through the second derivative maximum method.

\section{RESULTS}

\section{Screening study}

From a total of 1201 blood donors coming from Latin America, 23 were T. cruzi seropositive by the three serologic tests ,which corresponded to $1.91 \%$ of prevalence with respect to the total donor population. The Bolivian population with $16.03 \%$ (17 of 106; 95\% confidence interval [CI], 10.1-24.2) seropositivity had the highest prevalence (Table 1).

Parasite DNA was detected in peripheral WB by qPCR in 14 of the 23 seropositive donors, which corresponds to 60.86\% (95\% CI, 40.7-77.9) positivity in chronic patients (Table 1). The mean \pm SD Cq in these screening samples (GEB) was $34.35 \pm 2.87$, which implies a parasitic load of $0.39 \pm 0.2$ parasite equivalents $/ \mathrm{mL}$.

\section{Apheresis blood components study}

The qPCR study of the PLTs indicated that all samples amplified with $100 \%$ positivity in all the extractions (18 of 18) and $100 \%$ positivity in all replicates performed (54 of
54), with a significant mean \pm SD parasitemia of $5.33 \pm 6.12$ parasite equivalents/mL. In contrast, parasitemia was undetectable in all extract from plasma samples with $0 \%$ positivity in all the extractions ( 0 of 18 ) for parasite DNA, with $0 \%$ of amplification in all replicates (0 of 54). The peripheral WB (basal sample) obtained on the day of plasma and PLT donation by apheresis showed that all samples were positive with a mean \pm SD parasitemia of $0.42 \pm 0.32$ parasite equivalents $/ \mathrm{mL}$ (Table 2), although not all the replicates amplified in five extractions (59 of 72 replicates amplified by qPCR, 81.9\%; 95\% CI, 71.3-89.2).

\section{DISCUSSION}

Blood transfusion is the second most common mechanism for the transmission of T. cruzi in endemic areas and the first in nonendemic areas. The transfusion transmission risk of Chagas disease is thought to depend on multiple factors, including the level of parasitemia in the donor, the type of component transfused, and perhaps the strain of the parasite. ${ }^{17,18}$ In Spain, of the reported cases of $T$. cruzi transfusion transmission, all the patients were transfused with PLT concentrates and all the donors implicated in the donation originated from Chagas-endemic areas, ${ }^{4}$ three of the four donors from Bolivia and one from Brazil.

The serologic screening performed in our study in the Balearic Islands showed that $1.91 \%$ of the blood donors coming from Chagas-endemic areas were seropositive. The largest Latin American population living in the Balearic Islands originates from Argentina and is the Latin American group that donates blood most often; however, Bolivian donors have the highest positive detection rate for T. cruzi at $16.03 \%$. These results are consistent with the 2007 study performed in Catalonia, Spain, by Piron and colleagues, ${ }^{19}$ in which the seroprevalence of donors studied was $0.62 \%$, and the highest positive detection rate for T. cruzi (10.2\%) was in Bolivian blood donors.

The detection of $T$. cruzi by molecular methods, such as by PCR in blood samples, has high specificity and sensitivity. This technique has been successfully used in the diagnosis of acute chagasic disease for more than 15 years $;^{20}$ however, due to low parasitemia, the sensitivity and reproducibility of the technique decreases in chronic patients. The sensitivities reported in this group of 


\begin{tabular}{|l}
\hline \multicolumn{7}{|c|}{ TABLE 2. Peripheral WB and apheresis blood components from T. cruzi-seropositive blood donors and } \\
quantification results of parasitic load obtained by qPCR
\end{tabular}

patients are between 20 and $60 \%$ and depend on the conditions under which the technique is performed. ${ }^{15,21}$ In our study using a quantitative, validated, highly sensitive real-time PCR we found $60.86 \%$ (95\% CI, 40.7-77.9) positivity in 23 seropositive blood donors living in the Balearic Islands. This percentage is slightly higher than the $41 \%$ found in 2007 by Piron and coworkers ${ }^{15}$ in seropositive blood donors in Catalonia and similar to the $56.5 \%$ found in 2011 by Schijman and colleagues, ${ }^{21}$ using qPCR in chronic patients from endemic areas.

Low and oscillating parasitemias are described in chronic chagasic patients ${ }^{15,21}$ and are consistent with the results found in peripheral WB in our pool of seropositive donors, a group in which parasitemias were lower than 1 parasite equivalent $/ \mathrm{mL}$. In these samples it was observed that not all replicates amplified according to the low or nonexistence of parasites. Consequently, it is strongly recommended that replicates of DNA extractions and qPCR of $\mathrm{WB}$ are performed when a diagnosis is made to increase the possibility of finding positives results, especially when working with small volumes of samples.

Our extract from plasma results did not show any positive amplifications of the parasite DNA in any of the donor samples (0/6). By contrast, in the leukoreduced PLT concentrates, we found parasitic load values of $5.33 \pm 6.12$ parasite equivalents $/ \mathrm{mL}$, with a parasitic load range of 0.72 to 16.73 parasite equivalents $/ \mathrm{mL}$, more than five times greater than the parasitic load of the peripheral WB. The qPCR results were positive in all samples (6/6) and, interestingly, $100 \%$ of qPCR replicates of all samples studied $(54 / 54)$ were positives. It is possible that this percentage would decrease if no previous screening for the presence of parasite in peripheral $\mathrm{WB}$ was performed to select the studied individuals, as in the 2009 study carried out by Dzib and colleagues, ${ }^{22}$ using a conventional PCR of minicircle kDNA in blood components leukoreduced by centrifugation from 21 units of seropositive donors, which found $50 \%$ positivity in PLT concentrates. Results published in 2005 by Coronado and coworkers ${ }^{23}$ indicate that the circulating DNA belongs to living parasites and not to circulating DNA from the remains of dead parasites, because this is rapidly degraded. It would be interesting to study parasite viability to discriminate between viable and nonviable parasites.

Leukoreduction is considered by some authors to be a good method for reducing the T. cruzi transfusion transmission risk, but more detailed studies will be needed to confirm this theory. A report published by Hernández-Becerril and colleagues, ${ }^{24}$ in 2005, studied 70 PLT and RBC components leukoreduced from seropositive blood donors by conventional PCR and hemoculture. However, no parasitemia was detected by either of the methods; this factor could be attributed to the leukoreduction. Similarly, in 2006 Cardo and Asher ${ }^{9}$ reported a filtration assay study in which T. cruzi trypomastigotes spiked in plasma remained in the leukoreduction filters as a result of adherence. In 1995, MoraesSouza and coworkers, ${ }^{10}$ using leukoreduction filters in spiked human blood, showed that this process reduced the number of T. cruzi. By contrast, in our study, leukoreduction was not entirely effective at eliminating the parasitic load; in fact, the parasite loads detected in PLTs obtained by apheresis were higher than the parasite load in peripheral WB. A study performed by Dzib and coworkers ${ }^{22}$ in 2009 reported that leukoreduction by centrifugation did not eliminate T. cruzi from infected blood units. In fact, they found parasite DNA in RBCs, buffy coat, and PLT concentrates.

Certainly, the risk of $T$. cruzi transmission per infected blood product transfused is estimated to be from $10 \%$ to $25 \%$, with PLT products having a much higher risk than RBCs, frozen plasma, or cryoprecipitated products. ${ }^{4,9,25}$ In fact, there have been no reported cases of transfusion transmission through blood components that were frozen and stored at $-20^{\circ} \mathrm{C}$ or RBCs, even though a report published by Martin and coworkers ${ }^{26}$ in 2014 observed that T. cruzi in spiked human blood and in infected culture cells was able to survive long periods of storage at $+4^{\circ} \mathrm{C}$ and $-80^{\circ} \mathrm{C}$, suggesting that T. cruzi-infected blood or tissues stored under these conditions are potentially infectious.

The reason why we detected parasite DNA in PLTs, and not in plasma concentrates obtaining by apheresis from the same donor who was PCR positive in peripheral blood, could be related to the method that apheresis machines use to process WB. Basically, apheresis machines use centrifugation and filtration, the first as a method of 
separating and concentrating the selected blood component, through centrifugation that uses differences in specific gravity or density to separate and isolate blood components, and the second, filtration as a method for reducing the WBC count in PLT concentrates (some machines also use elutriation as an alternative to filtration). Filtration takes advantage of differences in particle size to separate and, as has already been stated, centrifugation is based on the specific gravity of blood cells. The trypomastigote form of $T$. cruzi has a size of 16 to $20 \mu \mathrm{m}^{18}$ and a specific gravity between PLTs and WBCs. ${ }^{27,28}$ According to these data, it is reasonable to think that, since PLTs are concentrated along with WBCs throughout the apheresis procedure, trypomastigotes are concentrated along with WBCs in the PLT fraction rather than in the plasma or RBC fraction. Therefore, the PLT fraction initially collected by apheresis contains WBCs, although it is leukoreduced during the procedure by elutriation or after the procedure by filtration. However, it is important to note that, despite leukoreduction (i.e., $\leq 1 \times 10^{6} / \mathrm{LWBCs}$ ) the level of residual WBCs in leukoreduced PLT concentrates could be up to 1 $\times 10^{6}$ per unit according to our national standards. Accordingly, if a considerable number of WBCs are still present in leukoreduced PLT products, it is logical to think that parasites can also remain.

Using laboratory techniques, in seropositive T. cruzi blood donors we have seen that PLTs are the component with the main parasitic load; therefore, it could explain what is observed in clinical cases of transfusion transmission of Chagas disease reported in the literature, in which PLT concentrates are the major component implicated in transmission. ${ }^{4-9,29}$ It would be challenging to investigate the role of new pathogen inactivation technologies, in reducing the parasite load in blood components, for example, PLT concentrates, obtained from seropositive T. cruzi donors, and to study whether they are effective in these samples, because so far they have only been studied experimentally in blood components spiked with T. cruzi. $^{30,31}$

\section{ACKNOWLEDGMENTS}

We acknowledge the blood donors for their participation on this study. The authors also thank Martin Hadley-Adams for his invaluable advice on the preparation of the manuscript.

\section{CONFLICT OF INTEREST}

The authors have disclosed no conflicts of interest.

\section{REFERENCES}

1. Pereira BI, Nazareth C, Malcata L, et al. Transfusion-transmitted protozoal infections: what is the risk in non-endemic countries? Acta Med Port 2011;24(Suppl 4):897-906.
2. Castro E. Chagas' disease: lessons from routine donation testing. Transfus Med 2009;19:16-23.

3. Bern C, Montgomery SP, Katz L, et al. Chagas disease and the US blood supply. Curr Opin Infect Dis 2008;21:476-82.

4. Benjamin RJ, Stramer SL, Leiby DA, et al. Trypanosoma cruzi infection in North America and Spain: evidence in support of transfusion transmission. Transfusion 2012;52:1913-21.

5. Young C, Losikoff P, Chawla A, et al. Transfusion-acquired Trypanosoma cruzi infection. Transfusion 2007;47:540-4.

6. Cimo PL, Luper WE, Scouros MA. Transfusion-associated Chagas' disease in Texas: report of a case. Tex Med 1993;89:48-50.

7. Grant IH, Gold JW, Wittner M, et al. Transfusion-associated acute Chagas disease acquired in the United States. Ann Intern Med 1989;111:849-51.

8. Nickerson P, Orr P, Schroeder ML, et al. Transfusion-associated Trypanosoma cruzi infection in a non-endemic area. Ann Intern Med 1989;111:851-3.

9. Cardo LJ, Asher L. Transfusion medicine illustrated. Electron micrographic study of the removal of Trypanosoma cruzi from blood products by leukodepletion filters. Transfusion 2006;46:1067-8.

10. Moraes-Souza H, Bordin JO, Bardossy L, et al. Prevention of transfusion-associated Chagas' disease: efficacy of white cell-reduction filters in removing Trypanosoma cruzi from infected blood. Transfusion 1995;35:723-6.

11. Kessler DA, Shi PA, Avecilla ST, et al. Results of lookback for Chagas disease since the inception of donor screening at New York Blood Center. Transfusion 2013;53:1083-7.

12. Blajchman MA. The clinical benefits of the leukoreduction of blood products. J Trauma 2006;60(Suppl 6):S83-90.

13. Duffy T, Cura CL, Ramirez JC, et al. Analytical performance of a multiplex real-time PCR assay using TaqMan probes for quantification of Trypanosoma cruzi satellite DNA in blood samples. PLoS Negl Trop Dis 2013;7:e2000

14. Riera C, Verges M, Iniesta L, et al. Identification of a Western blot pattern for the specific diagnosis of Trypanosoma cruzi infection in human sera. Am J Trop Med Hyg 2012; 86:412-6.

15. Piron M, Fisa R, Casamitjana N, et al. Development of a real-time PCR assay for Trypanosoma cruzi detection in blood samples. Acta Trop 2007;103:195-200.

16. Sabino EC, Lee TH, Montalvo L, et al. Antibody levels correlate with detection of Trypanosoma cruzi DNA by sensitive polymerase chain reaction assays in seropositive blood donors and possible resolution of infection over time. Transfusion 2013;53:1257-65.

17. Wendel S. Transfusion-transmitted Chagas' disease. Curr Opin Hematol 1998;5:406-11.

18. Rassi A Jr, Rassi A, Marin-Neto JA. Chagas disease. Lancet 2010;375:1388-402.

19. Piron M, Vergés M, Muñoz J. Seroprevalence of Trypanosoma cruzi infection in at-risk blood donors in Catalonia (Spain). Transfusion 2007;48:1862-8.

20. Wincker P, Telleria J, Bosseno MF, et al. PCR-based diagnosis for Chagas' disease in Bolivian children living in an active 
transmission area: comparison with conventional serological and parasitological diagnosis. Parasitology 1997;114:367-73.

21. Schijman AG, Bisio M, Orellana L, et al. International study to evaluate PCR methods for detection of Trypanosoma cruzi DNA in blood samples from Chagas disease patients. PLoS Negl Trop Dis 2011;5:e931.

22. Dzib D, Hernández VP, Ake BC, et al. Leukoreduction by centrifugation does not eliminate Trypanosoma cruzi from infected blood units. Vector Borne Zoonotic Dis 2009;9:235-41.

23. Coronado X, Ortiz S, Lastra O, et al. Instability of Trypanosoma cruzi DNA in blood lysates: importance for PCR DNAbased diagnosis. Mol Diagn 2005;9:35-40.

24. Hernández-Becerril N, Mejía AM, Ballinas-Verdugo MA, et al. Blood transfusion and iatrogenic risks in Mexico City. AntiTrypanosoma cruzi seroprevalence in 43,048 blood donors, evaluation of parasitemia, and electrocardiogram findings in seropositive. Mem Inst Oswaldo Cruz 2005;100:111-6.

25. Stramer SL, Hollinger FB, Katz LM, et al. Emerging infectious disease agents and their potential threat to transfusion safety. Transfusion 2009;49(Suppl 2):1S-29.
26. Martin DL, Goodhew B, Czaicki N, et al. Trypanosoma cruzi survival following cold storage: possible implications for tissue banking. PloS One 2014;9:e95398.

27. Amato NV, Matsubara L, Lanura PN. Avaliação do sistema quantitative buffy coat (QBC) no diagnóstico laboratorial da infecção pelo Trypanosoma cruzi: estudo em modelo experimental murino. Rev Soc Bra Med Trop 1996;29:59-61.

28. Ferreira CS, Bezerra RC, Pinheiro AA. Methylene blue vital staining for Trypanosoma cruzi trypomastigotes and epimastigotes. Rev Inst Med Trop 2006;48:347-9.

29. Leiby DA, Lenes BA, Tibbals MA, et al. Prospective evaluation of a patient with Trypanosoma cruzi infection transmitted by transfusion. N Engl J Med 1999;341:1237-9.

30. Tonnetti L, Thorp AM, Reddy HL, et al. Evaluating pathogen reduction of Trypanosoma cruzi with riboflavin and ultraviolet light for whole blood. Transfusion 2012;52:409-16.

31. Cardo LJ, Salata J, Mendez J, et al. Pathogen inactivation of Trypanosoma cruzi in plasma and platelet concentrates using riboflavin and ultraviolet light. Transfus Apher Sci 2007;37:131-7. 